
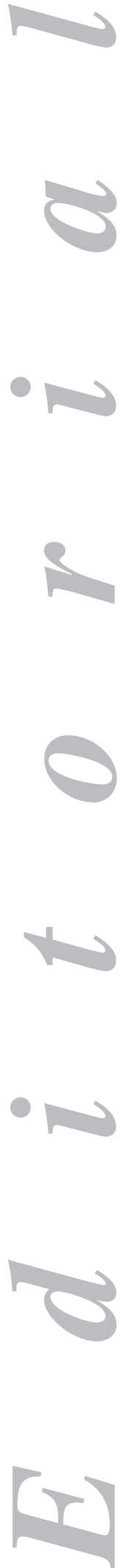

\section{Role of the peritoneum in the pathogenesis of acute pancreatitis-associated lung injury}

Acute pancreatitis is an unpredictable, potentially severe disease with an incidence of around 30 cases per 100,000 inhabitants and year $(1,2)$. In the USA, acute pancreatitis results in about 300,000 hospital admissions every year and a direct mortality of 3,200 deaths in that same period (3), which involves almost exclusively the $15-20 \%$ of patients who develop necrotic pancreatitis. Two periods of maximum mortality exist in the course of this disease - the first week, in which deaths most commonly result from multiple organ failure, and within a number of weeks, when infectious complications predominate (4).

The pathologic phenomenon that initiates pancreatitis is intrapancreatic activation of trypsinogen. This relevant piece of knowledge has provided insight into the pathogenesis of hereditary pancreatitis. Thus, a single-nucleotide mutation in the gene coding for cationic trypsinogen induces an amino acid substitution in the protein gene product, which may give rise to structural changes rendering the resulting trypsin extremely resistant to autolysis $(5,6)$ in an inappropriate time and place, since they allow for trypsin and protease co-localization (7) thus setting off the process of pancreatic self-digestion. Acinar cells undergo modifications (8), the expression of nuclear transcription factors such as NFKB becomes activated (9), and a complex proinflammatory cascade ensues, with synthesis and release of Th1 and Th2 cytokines, and phagocytic cell mobilization. Shortly afterwards this process goes beyond the anatomic limits of the pancreas and results in multisystem involvement, which is mostly responsible for the commonly serious outcome of this disease $(4,10)$.

The early stages of acute pancreatitis develop in a very short time within the pancreas, an organ whose deep anatomic location makes it virtually inaccessible for sample collection. Therefore, our knowledge on these early vital stages stems from a number of established experimental models that may not be extrapolated to human pancreatitis from an etiologic standpoint. The intraperitoneal injection of supramaximal doses of cerulein, a decapeptide analog of cholecystokinin, induces a mild, self-limited pancreatitis that is similar to human edematous pancreatitis (11). Severe necrotic pancreatitis is obtained by providing an experimental animal with a choline-deficient, ethionine-supplemented diet (12). The intraductal injection of taurocholate induces a fulminant pancreatitis that results in death in a few hours (13). Other models exist, but these have been most widely used.

Lung injury commonly develops early in acute pancreatitis, which may result in severe adult respiratory distress syndrome (ARDS). This disorder may be seen even in the cerulein-induced model $(11,14,15)$, and basically consists of increased permeability across the pulmonary endothelial barrier with hemorrhage, edema and interstitial infiltration by phagocytic cells (16), as well as oxygen desaturation (17). The human counterpart of such disturbances is well known (10), and its most obvious manifestation is an early development of pulmonary infiltrates, which may be detected in one fourth of patients (18) and definitely impairs prognosis (19).

Why does the lung become involved so much early and severely in acute pancreatitis? The inflammatory response triggered by pancreatitis rapidly reaches beyond pan- 
creatic limits, and pancreatic enzymes, proinflammatory cytokines, chemokines, growth factors, and other inflammation mediators escape from the pancreas into the blood and onto the peritoneum. It is a long-acknowledged fact that amylase, lipase and other pancreatic enzymes are present in increased concentrations in pancreatic ascites and pleural effusion secondary to pancreatitis (20). However, the presence of such enzymes does not imply pathogenicity, and elastase alone -maybe also trypsin- is seemingly responsible for lung damage through the activation of $\mathrm{NFKB}$ and the ensuing expression of the TNF $\alpha$ gene in the lung (21). Phospholipase $\mathrm{A}_{2}$ also plays a role in lung injury as it induces the release of arachidonic acid, thromboxanes, kinines, and platelet activating factor; the fact that this enzyme is of extrapancreatic origin is however somewhat enigmatic (20).

The role played by the peritoneum to extend this pathologic process is difficult to elicit, and procedures allowing the exclusion of phenomena directly depending on pancreas involvement should be put to good use therefore. This has been the goal of the paper by Mozo et al. in this issue of our journal (15). Their experimental model included the intraperitoneal injection of rat pancreas homogenate; one group of animals received enterokinase-activated homogenate, whereas the other group received a non-activated extract. A third -control- group received a saline solution. IL- $\beta 1$ concentration increased in both homogenate groups, more obviously so in the group receiving the activated form. However, only in the latter was observed a significantly more severe lung injury in comparison with the control group. The conclusion drawn by the authors is that peritoneal macrophages are the source of IL-1 $\beta$, and that this cytokine then reaches the lung and plays a role in injury mechanisms there. The role of peritoneal macrophages in triggering lung injury had already been demonstrated by Mikami et al. (22), who showed that peritoneal macrophage depletion reduced serum cytokines and lung damage in experimental pancreatitis in the rat.

An aspect left out by Mozo et al. is the role that the liver may play in the production and release of proinflammatory cytokines as a result of both Kupffer cell and sinusoid endothelium activation by products originating in the inflamed pancreas. This potential mechanism is actually supported by the fact that portal-cava shunting prevents increased pulmonary synthesis of prostacyclin and thromboxane $\mathrm{B}_{2}$, increased phospholipase $A_{2}$ concentrations, and increased superoxide dismutase activity (23) in experimental pancreatitis. In addition, Kupffer cell blockage using $\mathrm{ClGa}$ improves survival and reduces cytokine plasma concentrations in experimental models of severe pancreatitis (24).

The pathogenesis of acute pancreatitis-associated lung injury is very complex and remains to be fully understood; an in-depth analysis falls out of the scope of this editorial. The lung is invaded by a wide variety of proinflammatory mediators that in turn stimulate in situ production (25). Advances in the understanding of these mechanisms of injury will no doubt provide therapeutic strategies more selective and effective than those currently available. Such is the case of RANTES blocks using metRANTES, an antagonist of $\mathrm{C}-\mathrm{C}$ chemokine receptors (26), or $\mathrm{C}-\mathrm{x}-\mathrm{C}$ chemokine inhibition $(27,28)$. Other potential therapeutic goals include adhesion molecules VCAM-1 (29) and ICAM-1 (30), and macrophage migration inhibitory factor (MIF) (31). Interfering with NFKB (32) and platelet activating factor (PAF) expression may be of use in earlier stages of the disease, one of the latter's functions being macrophage activation (33); lexipafant, an antagonist of PAF, notably reduces lung injury in experimental pancreatitis, but clinical results are discouraging to this day (34) and seem to be restricted to patients who receive treatment within the first 48 hours of the disease (35). The therapeutic manipulation of both pro- and anti-inflammatory cytokines may also be of practical use (36).

The clinical applicability of these and other experimental findings remains in the future, since in most cases their preventive potential but not their therapeutic capability for an already existant pancreatitis is assessed. However, the contribution by Moro 
et al., who confirm the active role of the peritoneum and its resident macrophages in the pathogenesis of acute pancreatitis-associated lung injury, must be considered a further advance in the still long path towards eliciting the pathogenesis of this severe complication.

J. M. Ladero Quesada

\section{Service of Digestive Diseases. Hospital Clínico San Carlos.} Universidad Complutense. Madrid, Spain

\section{References}

1. Appelros S, Brogström A. Incidence, aetiology, and mortality rate of acute pancreatitis over 10 years in a defined urban population in Sweden. Br J Surg 1999; 86: 465-70.

2. Wilson C, Imrie CW. Changing patterns of incidence and mortality from acute pancreatitis in Scotland 19611985. Br J Surg 1990; 77: 731-4.

3. Sandler RS, Everhart JE, Donowitz M, Adams E, Cronin K, Goodman C, et al. The burden of selected digestive diseases in the United States. Gastroenterology 2002; 122: 1500-11.

4. Zhao X, Andersson R, Wang X, Dib M, Wang X. Acute pancreatitis-associated lung injury: patophysiological mechanisms and potential future therapies. Scand J Gastroenterol 2002; 37: 1351-8.

5. Whitcomb DC, Gorry MC, Preston RA, Furey W, Sossenheimer MJ, Ulrich CD, et al. Hereditary pancreatitis is caused by a mutation in the cationic trypsinogen gene. Nat Genet 1996; 14: 141-5.

6. Varallyay E, Pal G, Patthy A, Szilagyi L Graf L. Two mutations in rat trypsin confer resistance against autolysis. Biochem Biophys Res Commun 1998; 243: 56-60.

7. Steer ML. How and where does acute pancreatitis begin? Arch Surg 1992; 127: 1350-3.

8. Saluja AK, Bhagat L, Lee HS, Bhatia M, Frossard JL, Steer ML. Secretagogue-induced digestive enzyme activation and cell injury in rat pancreatic acini. Am J Physiol 1999; 276: G835-G842.

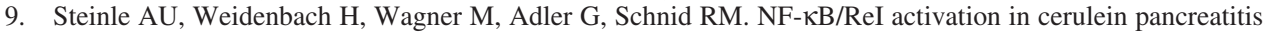
Gastroenterology 1999; 116: 420-30.

10. Pastor CM, Matthay MA, Frossard J-L. Pancreatitis-associated acute lung injury. New insights. Chest 2003; 124: 2341-51.

11. Lampel M, Kern H. Acute pancreatitis in the rat induced by excessive doses of a pancreatic secretagogue. Virch Arch Pathol Anat Histol 1989; 373: 1007-17.

12. Lombardi B, Estes L, Longnecker D. Acute hemorrhagic pancreatitis (massive necrosis) with fat necrosis induced in mice by DL-ethionine fed with a choline deficient diet. Am J Pathol 1975; 79: 465-80.

13. Senninger N. Bile-induced pancreatitis. Eur J Surg 1992; 24: 68-73.

14. Mozo G, del Olmo ML, Caro-Patón A, Reyes E, Manzano L, Belmonte A, et al. Afectación pulmonar y niveles de citocinas en un modelo de pancreatitis aguda experimental. Rev Esp Enferm Dig 2002; 94: 53-9.

15. Mozo G, del Olmo ML, Caro-Patón A, Reyes E, Manzano L, Belmonte A, et al. Pulmonary injuries and cytokine levels after the intraperitoneal administration of pancreatic homogenates in rats. Rev Esp Enferm Dig 2004; 96: 527-38.

16. Guice KS, Oldham KT, Jhonson KJ, Kunkel RG, Morganroth ML, Ward PA. Pancreatitis-induced acute lung injury. An ARSD model. Ann Surg 1988; 208: 371-8.

17. Yamanaka K, Saluja AK, Brown GE, Yamaguchi Y, Hofbauer B, Steer ML. Protective effect of prostaglandin E1 on acute lung injury of caerulein-induced acute pancreatitis in rats. Am J Physiol 1997; 272: G23-30.

18. Lankisch PG, Dröge M, Becher R. Pulmonary infiltrations: sign of severe acute pancreatitis. Int J Pancreatol 1996; 19: 113-5.

19. Talamini G, Uomo G, Pezzilli R, Rabitti PG, Billi P, Bassi C, et al. Serum creatinine and chest radiographs in the early assessment of acute pancreatitis. Am J Surg 1999; 177: 7-14.

20. Wilkinson MJ, Robson DK, Basran G. Pleural complications of acute pancreatitis: an autopsy study. Respir Med 1989; 83: 259-60.

21. Jaffray C, Yang J, Carter B, Mendez C, Norman J. Pancreatic elastase activates pulmonary nuclear factor $\mathrm{kB}$ and inhibitory $\mathrm{KB}$, mimicking pancreatitis-associated adult respiratory distress syndrome. Surgery $2000 ; 128$ : 225-31.

22. Mikami Y, Takeda K, Shibuya K, Qiu-Feng H, Shimamura H, Yamauchi J, et al. Do peritoneal macrophages play an essential role in the progression of acute pancreatitis in rats? Pancreas 2003; 27: 253-60.

23. Bardají CDM, Prats HN, Fernández-Cruz EGL, Roselló-Catafau J. Hepatic involvement in pancreatitis-induced lung damage. Am J Physiol 1996; 270: G6-G13.

24. Gloor B, Blinman TA, Rigberg DA, Todd KE, Lane JS, Hines OL, et al. Kupffer cell blockade reduces hepatic and systemic cytokine levels and lung injury in hemorrhagic pancreatitis in rats. Pancreas 2000; 21: 414-20.

25. Bhatia M, Moochhala S. Role of inflammatory mediators in the pathophysiology of acute respiratory distress syndrome. J Pathol 2004; 202: 145-56.

26. Bhatia M, Proudfoot AE, Wells TN, Christmas S, Neoptolemos JP, Slavin J. Treatment with Med-RANTES reduces lung injury in caerulein-induced pancreatitis. Br J Surg 2003; 90: 698-704. 
27. Osman MO, Kristensen JU, Jacobsen NO, Lausten SB, Deleuran B, Deleuran M, et al. A monoclonal interleukin-8 antibody (WS-4) inhibits cytokine response and acute lung injury in experimental severe acute necrotising pancreatitis in rabbits. Gut 1998; 43: 232-9.

28. Bhatia M, Brady M, Zagorski J, Christmas SE, Campbell F, Neoptolemos JP, et al. Treatment with neutralising antibody against cytokine induced neutrophil chemoattractant (CINC) protects rats against acute pancreatitis associated lung injury. Gut 2000; 47: 838-44.

29. Callicutt CS, Sabek O, Fukatsu K, Lundberg AH, Gaber L, Wilcox H, et al. Diminished lung injury with vascular adhesion molecule-1 blockade in choline-deficient ethionine diet-induced pancreatitis. Surgery 2003 133: $186-96$.

30. Werner J, Zgraggen K, Fernández del Castillo C, Lewandrowski KB, Compton CC, Warshaw AL. Specific therapy for local and systemic complications of acute pancreatitis with monoclonal antibodies against ICAM-1. Ann Surg 1999; 226: 834-42.

31. Sakai Y, Masamune A, Satoh A, Nishihira J, Yamagiwa T, Shimosegawa T. Macrophage migration inhibitory factor is a critical mediator of severe acute pancreatitis. Gastroenterology 2003; 124: 725-36.

32. Andersson R, Deng XM, Wang XD. The role of macrophage overactivation in the development of acute pancreatic injury in rats. Br J Surg 1997; 84: 775-80.

33. Katnik C, Nelson DJ. Platelet activating factor-induced increase in cytosolic calcium and transmembrane current in human macrophages. J Membr Biol 1993; 134: 213-24.

34. Johnson CD, Kingsnorth AN, Imrie CW, McMahon MJ, Neoptolemos JP, McKay C, et al. Double blind, randomised, placebo-controlled study of a platelet activating factor antagonist, lexipafant, in the treatment and prevention of organ failure in predicted severe acute pancreatitis. Gut 2001; 48: 62-9.

35. Kingsnorth AN. Early treatment with lexipafant, a platelet activating factor antagonist, reduces mortality in acute pancreatitis: A double blind, randomised, placebo controlled study. Gastroenterology 1997; 112 : A453.

36. Bruno MJ. Current insights into the pathogenesis of acute and chronic pancreatitis. Scand J Gastroenterol 2001; 36 (Supl. 234): 103-8

\section{Papel del peritoneo en la patogenia de la alteración pulmonar en la pancreatitis aguda}

La pancreatitis aguda es una enfermedad de curso imprevisible y potencialmente grave, cuya incidencia es de alrededor de 30 casos por 100.000 habitantes y año $(1,2)$. En los EE.UU. es causa de unos 300.000 ingresos hospitalarios al año con una mortalidad directa durante el mismo periodo de 3.200 defunciones (3), que inciden de forma casi exclusiva en el 15-20\% de enfermos que desarrollan una pancreatitis necrótica. Hay dos momentos evolutivos de máxima mortalidad: la primera semana, en la que las defunciones se deben mayoritariamente a fallo multiorgánico, y al cabo de varias semanas, periodo en el que juegan un papel protagonista las complicaciones infecciosas (4).

El fenómeno patológico que inicia la pancreatitis es la activación intrapancreática del tripsinógeno. Esta es una importante enseñanza que nos ha proporcionado el conocimiento de la patogenia de la pancreatitis hereditaria. Una mutación por cambio de un solo nucleótido en el gen del tripsinógeno catiónico induce a su vez una sustitución de un aminoácido en el producto proteico del gen, capaz de originar modificaciones estructurales que otorgan una resistencia anormalmente elevada de la tripsina naciente a la autolisis $(5,6)$ en un momento y lugar inadecuados porque permiten la co-localización de tripsina y proteasas (7) iniciando así el proceso de autodigestión del páncreas. Las células acinares sufren alteraciones (8), se activa la expresión de factores de transcripción nuclear como NFKB (9) y se desencadena una compleja cascada proinflamatoria con síntesis y liberación de citocinas Th1 y Th2 y movilización de células fagocitarias. Al poco tiempo, el proceso rebasa los límites anatómicos del páncreas y origina una afectación multisistémica que en gran medida es responsable de la grave evolución que con frecuencia sigue la enfermedad $(4,10)$. 
Las fases iniciales de la pancreatitis aguda se desarrollan en muy poco tiempo y en un órgano, el páncreas, cuya profunda situación anatómica le hace prácticamente inaccesible a la toma de muestras. Por lo tanto, los conocimientos que tenemos sobre estas primeras y trascendentales fases los hemos obtenido merced a diversos modelos experimentales bien establecidos, pero que no son extrapolables desde el punto de vista etiológico a la pancreatitis en el ser humano. La inyección intraperitoneal de dosis supramáximas de ceruleína, un decapéptido análogo de la colecistocinina, induce una pancreatitis leve y autolimitada análoga a la pancreatitis edematosa del ser humano (11). La forma grave, necrótica de pancreatitis se obtiene administrando al animal de experimentación una dieta deficiente en colina y suplementada con etionina (12). La inyección intraductal de taurocolato provoca una pancreatitis fulminante, mortal en pocas horas (13). Hay otros modelos, pero éstos han sido los más utilizados.

En el curso de la pancreatitis aguda es frecuente la aparición precoz de una afectación pulmonar que puede originar un grave síndrome de distrés respiratorio del adulto (SDRA). Incluso en el modelo inducido por la ceruleína se aprecia este trastorno $(11,14,15)$ que básicamente consiste en un aumento de la permeabilidad de la barrera endotelial pulmonar, con hemorragia, edema e infiltración de células fagocitarias en el intersticio (16) y desaturación de oxígeno (17). El correlato en clínica humana de estas alteraciones es bien conocido (10) y su manifestación más evidente es la aparición precoz de infiltrados pulmonares, que se detectan en la cuarta parte de los enfermos (18) y ensombrecen notablemente el pronóstico (19).

¿Por qué el pulmón resulta afectado de forma tan precoz y grave en la pancreatitis aguda? La respuesta inflamatoria desencadenada por la pancreatitis rebasa rápidamente los límites del páncreas, del que escapan a la sangre y al peritoneo enzimas pancreáticas, citocinas proinflamatorias, quimiocinas, factores de crecimiento y otros mediadores de la inflamación. Es un hecho conocido desde antiguo que amilasa, lipasa y otras enzimas pancreáticas están presentes en concentraciones elevadas en la ascitis pancreática y en el derrame pleural secundario a pancreatitis (20). Sin embargo, la mera presencia de una enzima no implica que sea patógena y parece que sólo la elastasa, y tal vez la tripsina, son capaces de inducir daño pulmonar a través de la activación de NFKB y la consiguiente expresión del gen TNF $\alpha$ en el pulmón (21). La fosfolipasa $\mathrm{A}_{2}$ también participa en el proceso lesivo pulmonar al inducir la liberación de ácido araquidónico, tromboxanos, cininas y factor activador de las plaquetas, aunque resulta algo enigmático que el origen de esta enzima sea extrapancreático (20).

El papel que juega el peritoneo en la extensión del proceso patológico es difícil de esclarecer y, para ello, hay que utilizar procedimientos que permitan hacer abstracción de los fenómenos dependientes de la afectación de la propia víscera pancreática. Este ha sido el objetivo del artículo de Mozo y cols. publicado en este número de la revista (15). El modelo experimental consistió en la inyección intraperitoneal de homogeneizado pancreático de ratas; a un grupo se le inyectó homogeneizado activado con enterocinasa y a otro un extracto no activado. Un tercer grupo, control de los otros dos, recibió suero salino. En ambos grupos tratados con homogeneizado se elevó la concentración de IL1 $\beta$, de forma más evidente en el que recibió el producto activado, y sólo en este se apreció un daño pulmonar significativamente más intenso que en el grupo control. La conclusión que extraen los autores es que los macrófagos peritoneales son fuente de IL1 $\beta$ y que esta citocina alcanza el pulmón y participa en los mecanismos lesionales a este nivel. El papel de los macrófagos peritoneales en el desencadenamiento del daño pulmonar ya había quedado demostrado por Mikami y cols. (22), quienes comprobaron que la depleción de macrófagos peritoneales reduce los niveles séricos de citocinas y el daño pulmonar en la pancreatitis experimental de la rata.

Un aspecto que no comentan Mozo y cols. es el papel que puede jugar el hígado en la producción y liberación de citocinas proinflamatorias como consecuencia de la activación de las células de Kupffer y del endotelio sinusoidal por efecto de los productos procedentes del páncreas inflamado. Este posible mecanismo se ve avalado por el hecho 
de que la creación de una anastomosis porto-cava evita el incremento de la síntesis pulmonar de prostaciclina y tromboxano $\mathrm{B}_{2}$, de la concentración de fosfolipasa $\mathrm{A}_{2}$ y de la actividad de superóxido dismutasa (23) en la pancreatitis experimental. Además, el bloqueo de las células de Kupffer con ClGa mejora la supervivencia y reduce las concentraciones plasmáticas de citocinas en modelos experimentales de pancreatitis grave (24).

La patogenia del daño pulmonar en la pancreatitis aguda es muy compleja y todavía no está bien esclarecida, y su análisis detenido queda fuera de los objetivos de este editorial. El pulmón es invadido por una gran variedad de mediadores proinflamatorios que a su vez estimulan su producción in situ (25). Los avances en el conocimiento de estos mecanismos lesionales proporcionará sin duda estrategias terapéuticas más selectivas y eficaces que las actualmente disponibles. Es el caso del bloqueo de RANTES con metRANTES, un antagonista de los receptores de quimiocinas C-C (26) o de la inhibición de quimiocinas $\mathrm{C}-\mathrm{x}-\mathrm{C}(27,28)$. Otros objetivos terapéuticos potenciales son las moléculas de adhesión VCAM-1 (29) e ICAM-1 (30) y el factor inhibidor de la migración de los macrófagos (MIF) (31). En fases más precoces puede ser útil interferir con la expresión del NFאB (32) y del factor activador de las plaquetas (PAF), entre cuyas funciones figura la activación de los macrófagos (33); lexipafant, un antagonista de PAF, reduce notablemente el daño pulmonar en la pancreatitis experimental, pero los resultados clínicos obtenidos hasta la actualidad son poco estimulantes (34) y parecen limitarse a aquellos casos que reciben el tratamiento durante las primeras 48 horas de la enfermedad (35). La manipulación terapéutica de las citocinas pro y antiinflamatorias también puede encontrar aplicación práctica (36).

La aplicabilidad clínica de estos y otros hallazgos experimentales aún debe esperar algún tiempo, ya que en la mayoría de los casos se valora su potencial preventivo, pero no la capacidad terapéutica una vez desencadenada la pancreatitis. No obstante, la aportación de Moro y cols. confirmando el papel activo del peritoneo y sus macrófagos residentes en la patogenia del daño pulmonar en la pancreatitis aguda debe ser valorada como un avance más en el todavía largo camino hacia el esclarecimiento de la patogenia de esta grave complicación.

J. M. Ladero Quesada

Servicio de Aparato Digestivo. Hospital Clínico San Carlos. Universidad Complutense. Madrid 